\title{
Possible Isotopic Fractionation Effects in Material Sputtered From Minerals
}

\author{
P. K. HafF, C. C. Watson, and T. A. Tombrello \\ W. K. Kellogg Radiation Laboratory, California Institute of Technology, Pasadena, California 91125
}

\begin{abstract}
We discuss in detail a model which makes definite predictions for the fractionation of isotopes in sputtered material. The fractionation patterns can be nonlinear, and the pattern for a particular set of isotopes depends on the chemical matrix within which those isotopes are contained. Calculations are presented for all nonmonoisotopic elements contained in the minerals perovskite, anorthite, ackermanite, enstatite, and troilite. All isotopes are fractionated at the level of approximately $4-6 \%$ per atomic mass unit. $\mathbf{O}$ is always positively fractionated (heavier isotopes sputtered preferentially), and heavier elements are generally negatively fractionated (lighter isotopes sputtered preferentially). The value of $\delta\left({ }^{18} \mathrm{O}:{ }^{16} \mathrm{O}\right)$ is always less by about $1.8 \%$ than a linear extrapolation based upon the calculated $\delta\left({ }^{17} \mathrm{O}:{ }^{16} \mathrm{O}\right)$ value would suggest. The phenomenon of both negative and positive fractionation patterns from a single target mineral can be used to make an experimental test of the proposed model.
\end{abstract}

Recent experiments [Russell et al., 1980] and theoretical models [Watson and Haff, 1980; Watson, 1980] have addressed the question of isotopic fractionation of surfaces that have been exposed to fluxes of low-energy (keV) ions. Earlier work has tended to center mainly on chemical composition changes produced by ion bombardment [Liau et al., 1977] since several convenient techniques (e.g., Rutherford backscattering) exist to analyze the near-surface composition as a function of depth. Theoretical studies of these processes, e.g., that of Haff and Switkowski [1976], always must prescribe quantitatively the differences in chemical binding energies between atomic species in order to predict the degree of chemical fractionation. In experiments involving ion sputtering of isotopic components of a single element the experimenter is faced with the complexities imposed by the small size of the expected effects, but the theoretical analysis of the sputtering process and the determination of relative sputtering yields for a given suite of isotopes is made correspondingly easier.

On the basis of an original motivation to construct a sputtering theory of binary and more complicated materials, which could be tested against experiment, Watson [1980] and Watson and Haff [1980] developed a model that described the sharing of recoil energy among the components of a given target. The results of the investigation indicated that little fractionation was likely to occur if fractionation effects were due entirely to differences in the bulk recoil fluxes of constituent target atoms. The calculated fractionation $\delta_{44,40}$ owing to nonstoichometries in the internal fluxes alone was only $\sim-1 \%$ (parts per thousand) for ${ }^{44} \mathrm{Ca}$ with respect to ${ }^{40} \mathrm{Ca}$ in a mineral target. We here define

$$
\delta_{1,2} \equiv \frac{S_{1} / n_{1}}{S_{2} / n_{2}}-1
$$

where $S_{i}$ is the number of target atoms of type $i$ sputtered per incident projectile, and $n_{i}$ is the abundance of atoms of type $i$ at the surface of the unsputtered target. Thus we compare the composition of the sputtered material to the undisturbed target composition. However, by making the critical assumption that the atoms found in the extreme outer layer cannot participate in the collision cascade on an equal footing with the internally recoiling atoms, Watson [1980] arrived at an ex-

Copyright (c) 1981 by the American Geophysical Union. pression for the isotopic fractionation expected in the material sputtered by the collisional recoil process from a surface containing isotopes of species $k$ and $l$ (This expression for $\delta_{k, l}$ is the one given in Watson [1980]. It differs slightly from that found in Watson and Haff [1980], although numerical values are similar in the two cases. For equal cross sections, the two expressions are identical.)

$$
\delta_{k, l}=\frac{\bar{\gamma}_{k}}{\bar{\gamma}_{l}}-1
$$

where

$$
\bar{\gamma}_{k}=\frac{\sum_{i} n_{j} \sigma_{i k} \gamma_{t k}}{\sum_{i} n_{j} \sigma_{i k}}
$$

$n_{i}$ is the fractional abundance of isotope $i, \sigma_{i k}=\sigma_{i l}$ is the lowenergy collision cross section between atoms of type $i$ and type $k$, and $\gamma_{t k}$ is a function of the atomic masses:

$$
\gamma_{i k}=\frac{4 M_{i} M_{k}}{\left(M_{i}+M_{k}\right)^{2}}
$$

The sums are taken over all target atomic species, but $k$ and $l$ refer only to isotopes of a single chemical element.

We emphasize that (2) applies only to the material actually sputtered away from the target and not to the composition of the target surface subsequent to sputtering. Modification of the surface composition can be described by models that incorporate both preferential sputtering effects and subsurface diffusion processes, but such a project is beyond the scope of the work discussed here. The abundance factors $n_{j}$ appearing in (2) refer to the instantaneous atomic abundances. In general, these are not constant in time if $\delta \neq 0$, and thus $\delta=\delta(t)$. The results which are reported here, therefore, refer only to low dose experiments where a limited amount of material is sputtered from the sample.

To illustrate the results one would expect if fractionation occurs according to (2), we first specialize to several idealized cases. Consider a target composed of a single element, which in turn is composed of only two isotopic species, 1 and 2 . The total cross sections $\sigma_{i j}$ are all equal to a common value, hence 


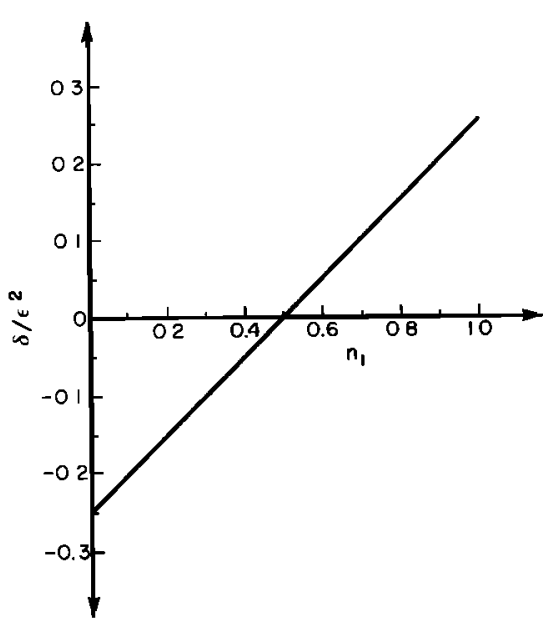

Fig. 1. The $\delta$ value of $(6)$ is plotted for a binary medium of two isotopic species $M_{1}, M_{2}$ with abundances $n_{1}, n_{2}$. The masses are related according to $M_{2}=M_{1}+\Delta M$, and $\varepsilon=\Delta M / M_{1}$ is taken to have a value of 0.1

$$
\delta_{1,2}=\frac{n_{1}+n_{2} \gamma_{12}}{n_{1} \gamma_{12}+n_{2}}-1
$$

We let the masses be $M_{1}$ and $M_{2}=M_{1}+\Delta M$ for species 1 and 2 , respectively, and furthermore we define $\Delta M / M_{1}=\varepsilon$. To illustrate the fractionation behavior for small mass differences we take $\varepsilon \ll 1$. Then $\gamma_{12} \approx \frac{1}{4} \varepsilon^{2}$

$$
\delta_{1,2} \approx \frac{1}{4} \varepsilon^{2}\left(n_{1}-n_{2}\right)
$$

For such a two-component system, the sign of the fractionation depends upon the abundance factors $n_{i}$ and not upon the mass values. Thus if $n_{1}>n_{2}$ in the surface layer, $\delta_{12}>0$ and species 1 is sputtered preferentially. Moreover, the fractionation effect is quadratic in the mass difference $\varepsilon$. For a mass increment of $\varepsilon=0.1$, (6) gives a maximum limiting value of $2.5 \%$ for the magnitude of the fractionation effect (see Figure 1).

The expression given by (2) and hence the result (6) arise from a detailed solution of the transport equation describing energy sharing among recoiling atoms [Watson, 1980; Watson and Haff, 1980]. A source of particles is created by primary collisions between the incident ion and a target atom. These recoiling atoms are typically of very low energy and interact much in the manner of hard spheres. For projectiles in the energy range of a few $\mathrm{keV}$ to a few hundred $\mathrm{keV}$, a description of the sputtering process based upon such a picture [Sigmund, 1969] is well established. Absolute sputtering yields (number of atoms ejected per incident ion) of many materials can be computed to an accuracy within a factor of 2 or 3, and, more importantly, the dependence of the sputtering yield on incident ion energy, mass, and charge can be reproduced adequately by the cascade theory for a wide variation in the parameters. Furthermore, the predicted $E^{-2}$ dependence of the yield on the energy of the sputtered particle has been independently verified by several investigators [Thompson, 1968; Weller and Tombrello, 1978].

These calculations have in each case been performed for a uniform distribution of scattering centers. In an actual sputtering experiment, however, the distribution of atoms fills only a half-space. The actual calculations are thus perturbation theory calculations, with the target surface introduced only at the final step as the boundary across which the sputtering flux should be taken. Sequential collision events such as those shown in Figure 2 are included in the calculation but cannot physically occur in the true target since the collision point is outside the target surface. Watson [1980] and Watson and Haff [1980] made an attempt to include effects introduced by the presence of a surface in a less rigorous way. They postulated that the atoms comprising the extreme outer surface layer of the target cannot participate fully in the recoil cascade, primarily because the geometry makes it difficult for them to transfer energy to subsequent atoms after they themselves have been struck. Sputtered particles are then imagined to derive from this passive surface layer as it absorbs energy from recoiling atoms deeper in the target. It is this model, which attempts to take account of the effects of a nonuniform (i.e., half-space) distribution of target atoms, that leads to the expression (2) for the fractionation.

The 'surface flux' model was adopted because the nonstoichiometric emission of isotope species expected from the bulk recoil flux alone was found to be much smaller than indicated by experiment. Thus Russell et al. [1980] found $\delta\left({ }^{44} \mathrm{Ca}:{ }^{40} \mathrm{Ca}\right)$ for material sputtered from a plagioclase target to be on the order of $\sim-20 \%$, while predictions based on nonstoichiometries in the bulk recoil flux indicated values no less than -1\%o [Watson and Haff, 1980]. On the other hand, the surface flux model is in adequate agreement with the data of Russell et al. [1980] for Ca fractionation in plagioclase and in fluorite. However, the peculiar fractionation patterns inherent in the model, as shown clearly by (6), which predicts (1) that the value and even the sign of $\delta$ depend upon the abundance factors of the target components and (2) that it is possible for the fractionation pattern to contain no linear term in the mass increment, have not yet been adequately tested experimentally. In the subsequent discussion we will clarify the physics behind the fractionation mechanism at issue here and suggest some further experiments designed explicitly to test the model.

We continue for a moment our discussion of a two-component medium. According to Watson and Haff [1980], the bulk flux of recoiling atoms is very nearly stoichiometric (except for extreme differences in target atom masses or for the very highest energy particles, which, however, constitute only a small portion of the total flux). This flux has an energy dependence of the form $A n_{i} / E^{2}$, for species $i$, where $A$ is a constant. For simplicity, imagine head-on collisions of these bulk

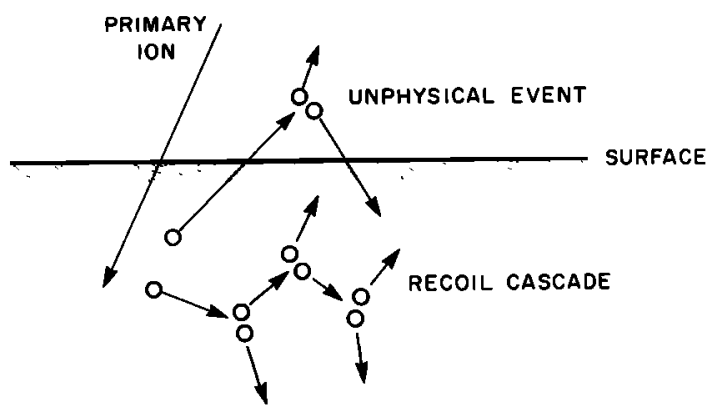

Fig. 2. This schematic picture shows the type of unphysical collision (taking place outside the solid) that is unavoidably included in most calculations of the sputtering yield. The surface flux model attempts to partly compensate for the errors introduced in such calculations by decoupling the extreme surface layer of the target from the recoil cascade, except for collisions that actually yield a sputtered particle. 
flux components with stationary surface atoms. The surface flux of type 1 atoms is then

$$
d G_{1} \propto n_{1} \gamma_{11} \mathrm{~A} \frac{n_{1}}{E^{2}} d E+n_{1} \gamma_{12} \mathrm{~A} \frac{n_{2}}{E^{2}} d E
$$

where the first term describes atoms of type 1 ejected by collision with atoms of type 1 , and the second term describes atoms of type 1 ejected by collision with atoms of type 2 . Equation (7) leads to the partial sputtering yields

$$
d S_{1} \propto n_{1}\left(n_{1}+\gamma_{12} n_{2}\right) d E / E^{2}
$$

and similarly

$$
d S_{2} \propto n_{2}\left(n_{2}+\gamma_{21} n_{1}\right) d E / E^{2}
$$

The fractionation of the sputtered material with respect to the bulk is therefore

$$
\delta_{1,2}=\frac{\int d S_{1} / \int d S_{2}}{n_{1} / n_{2}}-1
$$

which reduces to (5) upon substitution from (8) and (9). The $\gamma$ factors in (7) come from the kinematic limits on the maximum amount of energy that can be transferred in an elastic collision. The source of the fractionation in this model is therefore seen to arise from the energy transfer mismatch between the surface species and the bulk recoil flux; i.e., it is easier to transfer energy to a similar mass in a collision than to a much different mass. The effect is larger in the surface layer than it is internally because of the fact that surface atoms are allowed to interact with the cascade in one step only: there is no opportunity for the effect to be averaged away over many collisions. Equations similar to (8) and (9), and their generalization to polyatomic media, were derived by Watson [1980] and Watson and Haff [1980] without the simplifying assumption of head-on collisions used here. In the general case in which different chemical species are present in the target, the relative total collision cross sections that describe scattering of the various distinct pairs of atoms also appear in the expression for $\delta$, as in ((2). Equations (2) and (3) are applicable to realistic, chemically complex targets and are utilized in this paper.

These remarks apply to isotopic components of a single element. If more than one chemical species is present, fractionation of one chemical species from another will in general occur, but its magnitude is determined more by details of the target chemistry than by the atomic masses and abundances. However, expression (2) for the fractionation applies to any target, regardless of its chemical composition, as long as it is only the fractionation among isotopic components of a single chemical element that is desired. Moreover, since members of any isotopic suite are ejected from the surface by means of collisions with all the atomic species in the target, it is clear that the fractionation pattern for these isotopes can depend strongly on the mass of each kind of target atom. It is this feature that we feel will provide the most convincing test of the validity of the fractionation model described here.

As an illustration of the effect of this 'background' mass $M_{3}$ upon the fractionation pattern of a particular pair of isotopes 1 and 2 with masses $M_{1}$ and $M_{2}\left(M_{1}<M_{2}\right.$, say), we consider a target composed principally of type 3 background atoms with abundance $n_{3}$, but which contains a small proportion of the isotopic species 1 and 2 so that $n_{1}+n_{2} \ll n_{3}$. Type 3 atoms need not be isotopes of the same element as type 1 and type 2

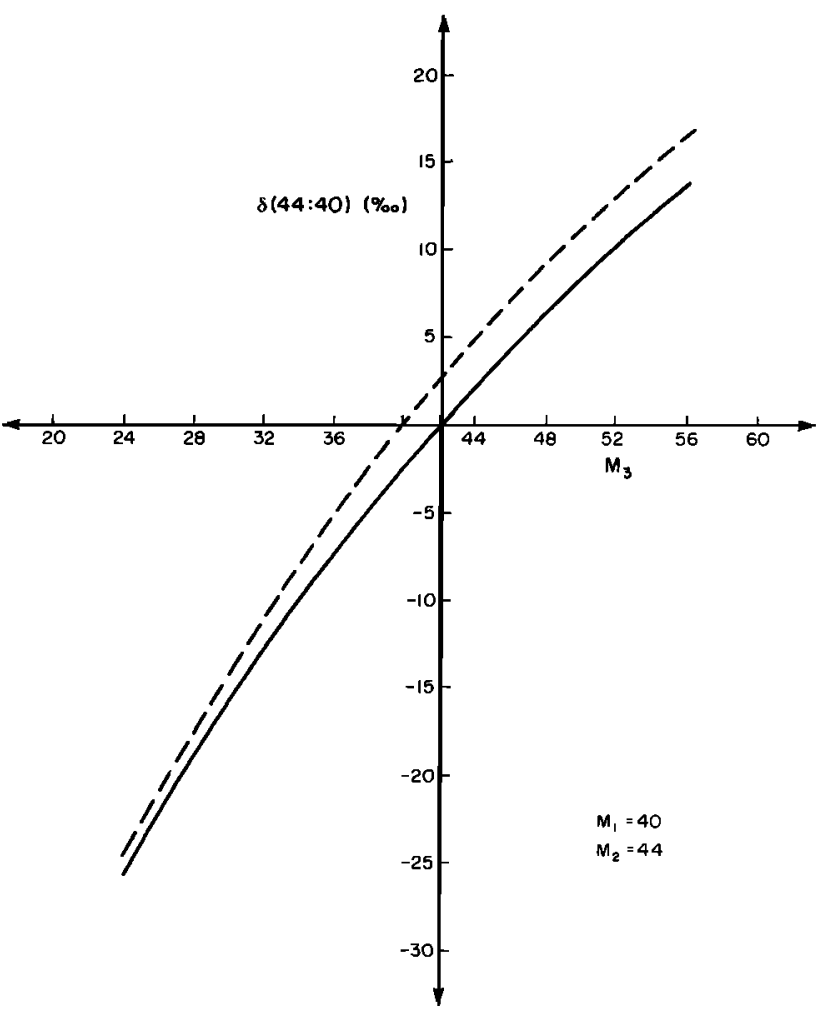

Fig. 3. This figure shows the fractionation predicted for a hypothetical target containing traces of mass 40 and 44 imbedded in a 'background' matrix of atomic mass $\boldsymbol{M}_{3}$. The dashed curve shows the fractionation calculated in the linear approximation (12), and the solid curve gives the exact fractionation calculated according to (2). The characteristic changeover from negative to positive fractionation as a function of the background mass is clearly shown. Note that the nonlinearity in the fractionation of the isotopes $\mathbf{4 0}$ and $\mathbf{4 4}$ is not given by the deviation from a straight line of the curves shown here, since only the $\delta$ values for a single pair are represented.

atoms. The last inequality is not necessary for analysis but it leads to a clear picture of the fractionation process. Essentially all atoms that are ejected will arise from collisions with type 3 atoms. Thus when $M_{3}<M_{1}$ we expect species 1 to be preferentially sputtered so that $\delta_{2,1}<0$, but if $M_{2}<M_{3}$, then $\delta_{2,1}>$ 0 . For the case $M_{1}<M_{3}<M_{2}$ the fractionation will be small, and it will vanish for some value of $M_{3}$ in this range. For this case we find from (2)

$$
\delta_{2,1} \approx \frac{\gamma_{23}}{\gamma_{13}}-1
$$

since the cross sections $\sigma_{23}$ and $\sigma_{13}$ are equal. The fractionation in this case is independent of the abundances of the isotopes in question, as long as they are much less than unity. If $M_{2}=$ $M_{1}+\Delta M$ and $\varepsilon \equiv \Delta M / M_{1}$, then for $\varepsilon \ll 1$,

$$
\delta_{2,1} \approx-\varepsilon\left(\frac{1-M_{3} / M_{1}}{1+M_{3} / M_{1}}\right)
$$

so that the fractionation is linear, with $-\varepsilon \leq \delta_{2,1} \leq \varepsilon$. Figure 3 shows $\delta_{2,1}$ as a function of $M_{3}$ for the particular choice $\varepsilon=0.1$, $M_{1}=40$ (and $M_{2}=44$ ). Also shown is $\delta_{2,1}$ evaluated according to (11), so that an expansion in powers of $\varepsilon$ is avoided. The two curves differ significantly in the region $M_{3} \approx M_{1}$, but they both illustrate the fact that the magnitude and sign of $\delta_{2,1}$ can be drastically affected by the choice of the chemical matrix in 


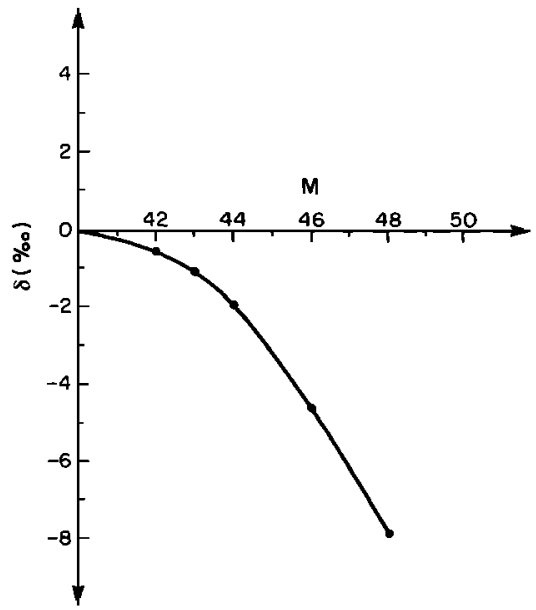

Fig. 4. The curve shows the predicted fractionation in a pure $\mathrm{Ca}$ metal target containing terrestrial abundances of the isotopes. The deviation from linearity is due to higher order terms in the mass increment $\varepsilon$. The leading term is linear.

which the isotopic species of interest are imbedded. If for simplicity we take $n_{1}, n_{2}$, and $n_{3}$ to refer to isotopes of a single element, then in the special (but unlikely) case $n_{1}=n_{2}=n_{3}=1 / 3$ we find $\delta_{2,1}=\frac{1}{4} \varepsilon^{2}$ and $\delta_{3,1}=0$ for $M_{2}=M_{1}+1$ and $M_{3}=M_{1}+$ 2 with $\varepsilon=1 / M_{1} \ll 1$. Here the middle mass isotope $M_{2}$ is better coupled to the underlying recoil cascade than are either of the end members.

Although, with the exception of special cases (see (6) and the above paragraph), $\delta$ is proportional to the mass increment $\varepsilon$ for small $\varepsilon$, if this quantity is not small, then $\delta$ will depend upon the increment in a more complicated way. We do not need to appeal to an expansion in $\varepsilon$ to see this effect, since within the model, (2) is exact. Figure 4 gives an illustration of nonlinearities in the fractionation, owing to violation of the condition $\varepsilon \ll 1$, for a pure $\mathrm{Ca}$ target containing the terrestrial abundances of ${ }^{40} \mathrm{Ca},{ }^{42} \mathrm{Ca},{ }^{43} \mathrm{Ca},{ }^{44} \mathrm{Ca},{ }^{46} \mathrm{Ca}$, and ${ }^{48} \mathrm{Ca}$. The curve was calculated from (2). The $\delta$ values with respect to ${ }^{40} \mathrm{Ca}$ are all negative, since the dominant abundance of ${ }^{40} \mathrm{Ca}$ leads to preferential ejection of the lighter isotopes.

We turn now to more interesting targets, taking as a first example $\mathrm{SiO}_{2}$. Because for the light elements stable light isotopes are generally more abundant than heavier isotopes, we would expect for a pure Si target $\left(n_{28}=0.9217, n_{29}=0.0471\right.$, $n_{30}=0.0312$ ) that $\delta_{29,28}$ and $\delta_{30,28}$ would be negative, while for a pure (solid) $O$ target $\left(n_{16}=0.99759, n_{17}=0.00037, n_{18}=\right.$ $0.00204), \delta_{17,16}$ and $\delta_{18,16}$ would also be negative. However, for the $\mathrm{SiO}_{2}$ target, the qualitative conclusions reached for the sign of the $\delta$ values above are no longer all necessarily true. First, although the addition of $\mathrm{O}$ to a $\mathrm{Si}$ target would tend to make the $\delta_{29,28}$ and $\delta_{30,28}$ values even more negative, the addition of $\mathrm{Si}$ to an $\mathrm{O}$ target would have the opposite effect on $\delta_{17,16}$ and $\delta_{18,16}$ and could even make them positive. There is the additional complication of the cross-section values to be used (see (3)). When two or more chemically distinct species are present in the target with nonnegligible abundances, the total low-energy scattering cross sections help to determine the fractionation values. The $\sigma_{i j}$ enter the calculation because the collision probabilities determining the coupling of the surface layer to the bulk depend on the product of abundance times cross section. Because of the structure of (3), one of these cross sections may be chosen arbitrarily, i.e., we are interested in the relative, not absolute, sputtering rates. Con- sequently, the fractionation values depend only upon the ratios of cross sections and not on their magnitudes. When a target is composed of isotopes of one element only, or where collisions between such isotopes and one other distinct element are the only collisions which are important, then the ratios of all relevant cross sections become unity, as is the case in the above examples, and $\delta$ then depends on the masses and abundances alone.

To begin with, we illustrate the fractionation expected for both $\mathrm{Si}$ and $\mathrm{O}$ in $\mathrm{SiO}_{2}$, with the values (taken from a BornMayer model of the atom) $\sigma_{\mathrm{Si} \rightarrow \mathrm{Si}}\left(=\sigma_{28,28}=\sigma_{28,29}\right.$, etc. $)=5.91$ $\AA^{2}, \sigma_{\mathrm{Si} \rightarrow 0}\left(=\sigma_{28,16}=\sigma_{28,17}=\sigma_{29,16}\right.$, etc. $)=5.14 \AA^{2}$, and $\sigma_{\mathrm{O} \rightarrow \mathrm{O}}=$ $4.43 \AA^{2}$. (Typical recoil velocities are so low $(1-10 \mathrm{eV}$ ) that highly screened neutral-atom cross sections are appropriate.) We find from (2)

$$
\delta_{17,16}=+4.9 \%
$$

and

$$
\delta_{18,16}=+8.0 \%
$$

so that the deviation from linearity is $1.8 \%$. The Si fractionation values are

$$
\delta_{29,28}=-6.4 \%_{0}
$$

and

$$
\delta_{30,28}=-13.0 \%
$$

which is essentially a linear relationship. The important point though is that the trend of the fractionation corresponds to preferential emission of heavy isotopes for one chemical element $(O)$ and to preferential emission of light isotopes for another ( $\mathrm{Si})$.

The above values are dependent upon the cross sections for scattering of $\mathrm{Si}$ and $\mathrm{O}$ atoms. To get an idea of the sensitivity of $\delta$ to the choice of cross section, we arbitrarily interchange the $\mathrm{Si} \rightarrow \mathrm{Si}$ scattering cross section with the $\mathrm{O} \rightarrow \mathrm{O}$ scattering cross section in the evaluation of $\delta$, i.e., $\delta_{\mathrm{Si} \rightarrow \mathrm{Si}} \rightarrow 4.43 \AA^{2}, \sigma_{\mathrm{O} \rightarrow 0}$ $\rightarrow 5.91 \AA^{2}$, but $\sigma_{\mathrm{Si} \rightarrow 0}=5.14 \AA^{2}$ as before. Then

$$
\delta_{17,16}=3.9 \%
$$

and

$$
\delta_{18.16}=5.9 \%
$$

with roughly the same amount of nonlinearity as above, while

$$
\delta_{29,28}=-6.9 \%
$$

and

$$
\delta_{30,28}=-14.1 \%
$$

still almost linear. Finally, in the case that all cross sections are set equal to each other, we find

$$
\delta_{17,16}=4.1 \% 0 \quad \delta_{18,16}=6.4 \% 0 \quad \delta_{29,28}=-6.5 \%
$$

and

$$
\delta_{30,28}=-13.2 \%
$$

Figure 5 summarizes the fractionation patterns for these three choices of the cross sections.

The dependence of $\delta$ upon the (generally not well known) collision cross sections is a fact one must live with. It makes a reliable calculation of expected $\delta$ values more difficult. Still, the patterns illustrated in Figure 5 are relatively stable against 


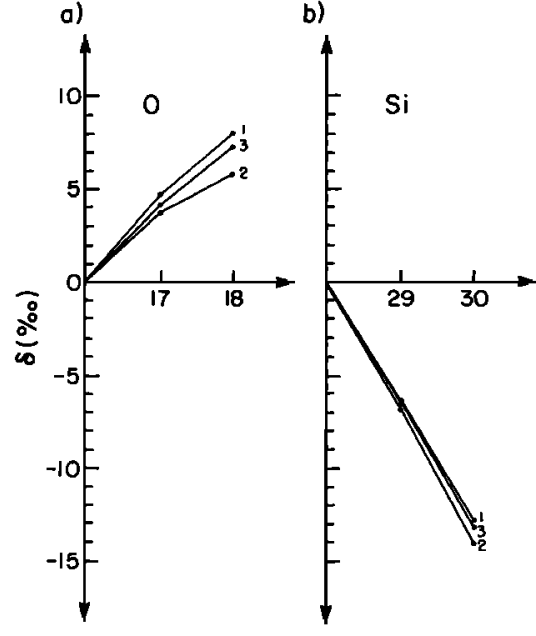

Fig. 5. The three curves in (a) and (b) summarize the fractionation pattern for three different choices of the relative scattering cross sections in an $\mathrm{SiO}_{2}$ target. In (a), curve 1 corresponds to $\sigma_{\mathrm{O}-\mathrm{O}} / \sigma_{\mathrm{S}_{1}-\mathrm{O}}=$ 0.86 , curve 2 corresponds to $\sigma_{0-O} / s_{\mathrm{s}-0}=1.16$, and curve 3 corresponds to $\sigma_{\mathrm{O}-\mathrm{O}} / \sigma_{\mathrm{S}_{1}-\mathrm{O}}=1.0$. In $(b)$, curve 1 corresponds to $\sigma_{\mathrm{S}_{1}-\mathrm{Si}} / \sigma_{\mathrm{Si}-}$ $\mathrm{o}=1.15$, curve 2 corresponds to $\sigma_{\mathrm{Si}-\mathrm{S}_{1}} / \sigma_{\mathrm{S} 1-\mathrm{O}}=0.87$, and curve 3 corresponds to $\sigma_{\mathrm{Si}-\mathrm{S} /} / \mathrm{si-O}=1.0$.

variations in the $\sigma_{\mathrm{j} j}$. One reason is that only the relative values of the cross section are required in (3).

In order to illustrate the application of the above results to particular targets, we give some examples of predictions based upon (2) as applied to selected high temperature condensate minerals. Mineral targets were chosen for several reasons. The most complete characterization of isotopic fractionation effects induced by sputtering was recently carried out on plagioclase and fluorite targets [Russell et al., 1980]. The present model was developed in part to try to understand these results. In earlier work the question of isotopic fractionation of mineral components during sputtering was addressed by $S$ witkowski et al. [1977] to try to account for the observed abundance of $\mathrm{Si}$ and $\mathrm{O}$ isotopes in lunar fines. More recently Clayton [1981] has raised the question of whether sputtering of interstellar grains could be the source of certain isotopic anomalies observed in meteoritic inclusions. In the examples illustrated below we do not endeavor to come to grips with the problem of the likeliness of sputtering as a significant isotopic fractionating agent in pre-solar system history, but it is nevertheless interesting to see how appropriate mineral phases [Grossman, 1972] would be expected to fractionate in the present model. By presenting these examples, we also gain a clearer idea of the magnitude of the effects that might be expected in an actual experimental test of the model.

Figures 6-10 show the predicted fractionation patterns for all sets of isotopes in, respectively, perovskite $\left(\mathrm{CaTiO}_{3}\right)$, plagioclase (anorthite) $\left(\mathrm{CaAl}_{2} \mathrm{Si}_{2} \mathrm{O}_{8}\right)$, melilite (akermanite $\left(\mathrm{Ca}_{2} \mathrm{MgSi}_{2} \mathrm{O}_{7}\right)$ ), enstatite $\left(\mathrm{MgSiO}_{3}\right)$, and troilite (FeS). To be definite we have set all cross sections equal. The patterns exhibit several interesting features. Perhaps most striking is the fact that the fractionation effect in one mineral looks essentially the same as in any other mineral containing the same kinds of atoms. The same basic pattern is also encountered in a number of other minerals not illustrated here: corundum $\left(\mathrm{Al}_{2} \mathrm{O}_{3}\right)$, magnetite $\left(\mathrm{Fe}_{3} \mathrm{O}_{4}\right)$, rutile $\left(\mathrm{TiO}_{2}\right)$, eskolaite $\left(\mathrm{Cr}_{2} \mathrm{O}_{3}\right)$, various spinels $\left(\mathrm{FeAl}_{2} \mathrm{O}_{4}, \mathrm{ZnAl}_{2} \mathrm{O}_{4}, \mathrm{MnAl}_{2} \mathrm{O}_{4}, \mathrm{MgAl}_{2} \mathrm{O}_{4}\right)$, gehlenite $\left(\mathrm{Ca}_{2} \mathrm{Al}_{2} \mathrm{SiO}_{7}\right)$, hibonite $\left(\mathrm{CaAl}_{12} \mathrm{O}_{19}\right)$, fluorite $\left(\mathrm{CaF}_{2}\right)$, albite $\left(\mathrm{NaAlSi}_{3} \mathrm{O}_{8}\right)$, diopside $\left(\mathrm{CaMgSi}_{2} \mathrm{O}_{6}\right)$, and forsterite $\left(\mathrm{Mg}_{2} \mathrm{SiO}_{4}\right)$. In each case $\mathrm{O}$ is fractionated to a level of roughly 4-6\% per mass unit, with a slight $(\sim 1.8 \%$ o $)$ but remarkably constant nonlinearity to be discussed below. The $O$ curves correspond to positive fractionation, and the reason is the

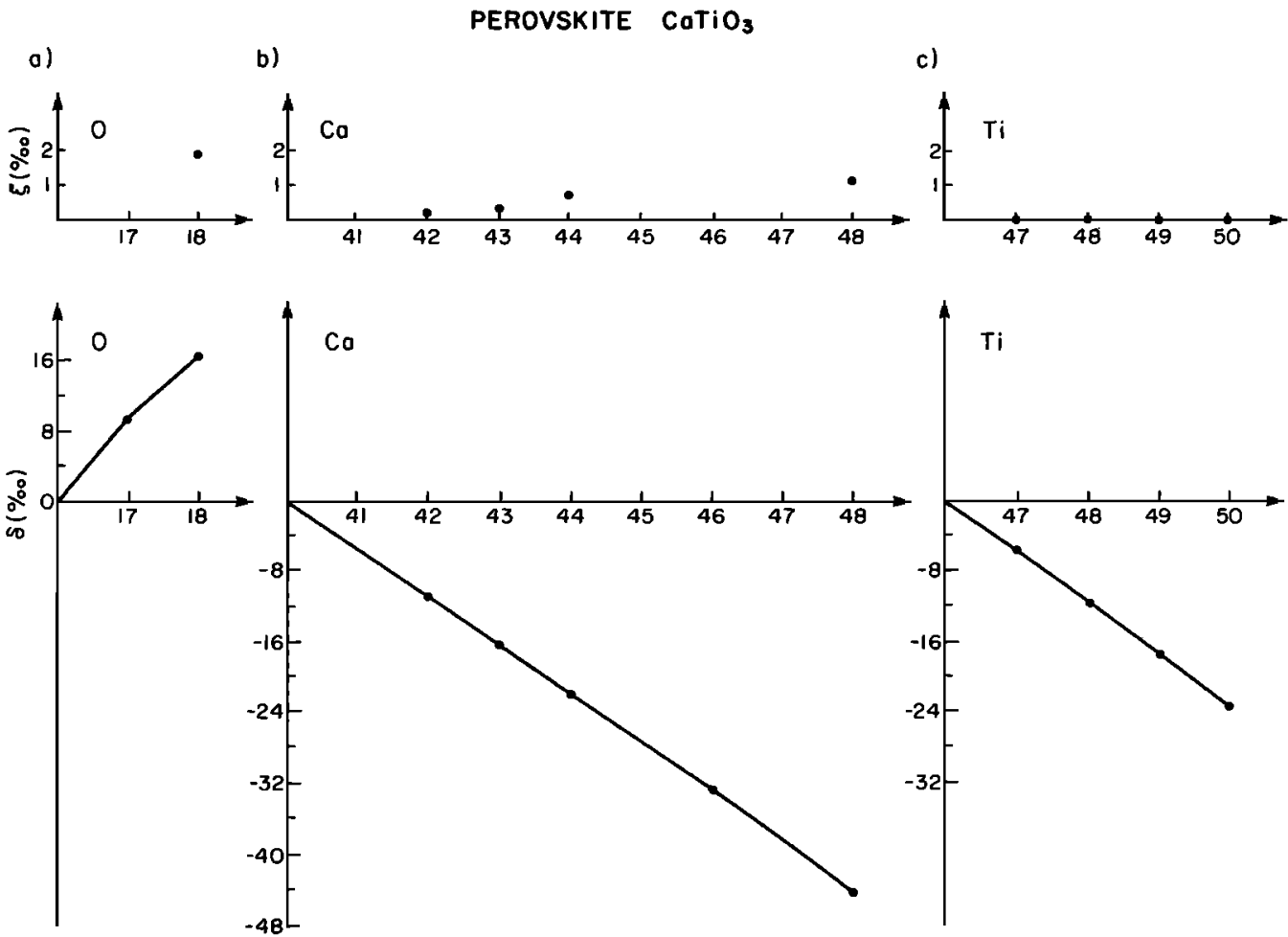

Fig. 6. In (a), (b) and (c) the lower panels show the fractionation $\delta$ for $\mathrm{O}, \mathrm{Ca}$, and $\mathrm{Ti}$, respectively, in perovskite, $\mathrm{CaTiO}_{3}$. The upper panels in each case show the deviation from linearity as defined by (13). 

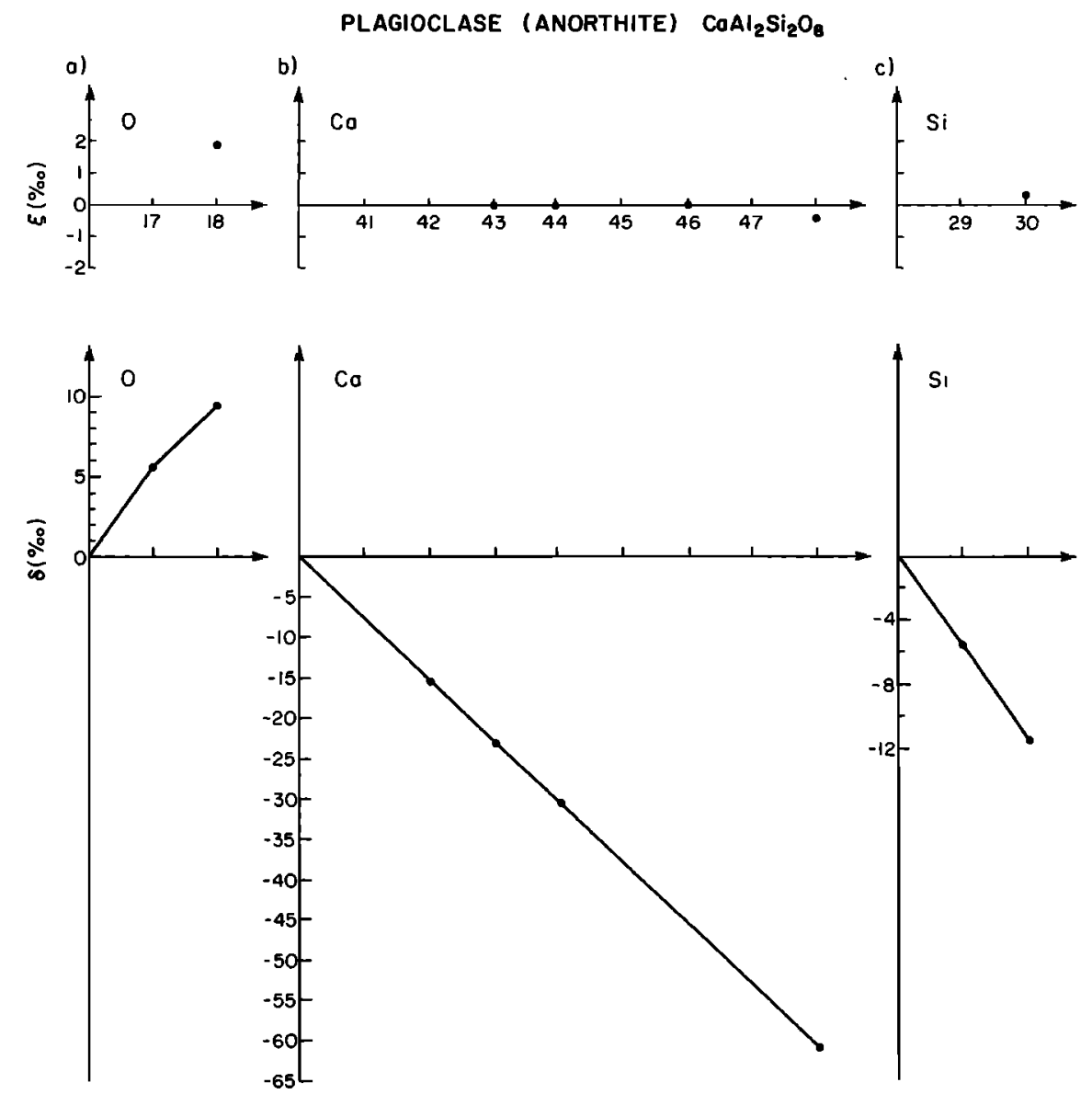

Fig. 7. In (a), (b), and (c) the lower panels show the fractionation $\delta$ for $\mathrm{O}, \mathrm{Ca}$, and $\mathrm{Si}$, respectively, in anorthite, $\mathrm{CaAl}_{2} \mathrm{Si}_{2} \mathrm{O}_{8}$. The upper panels in each case show the deviation from linearity as defined by (13).
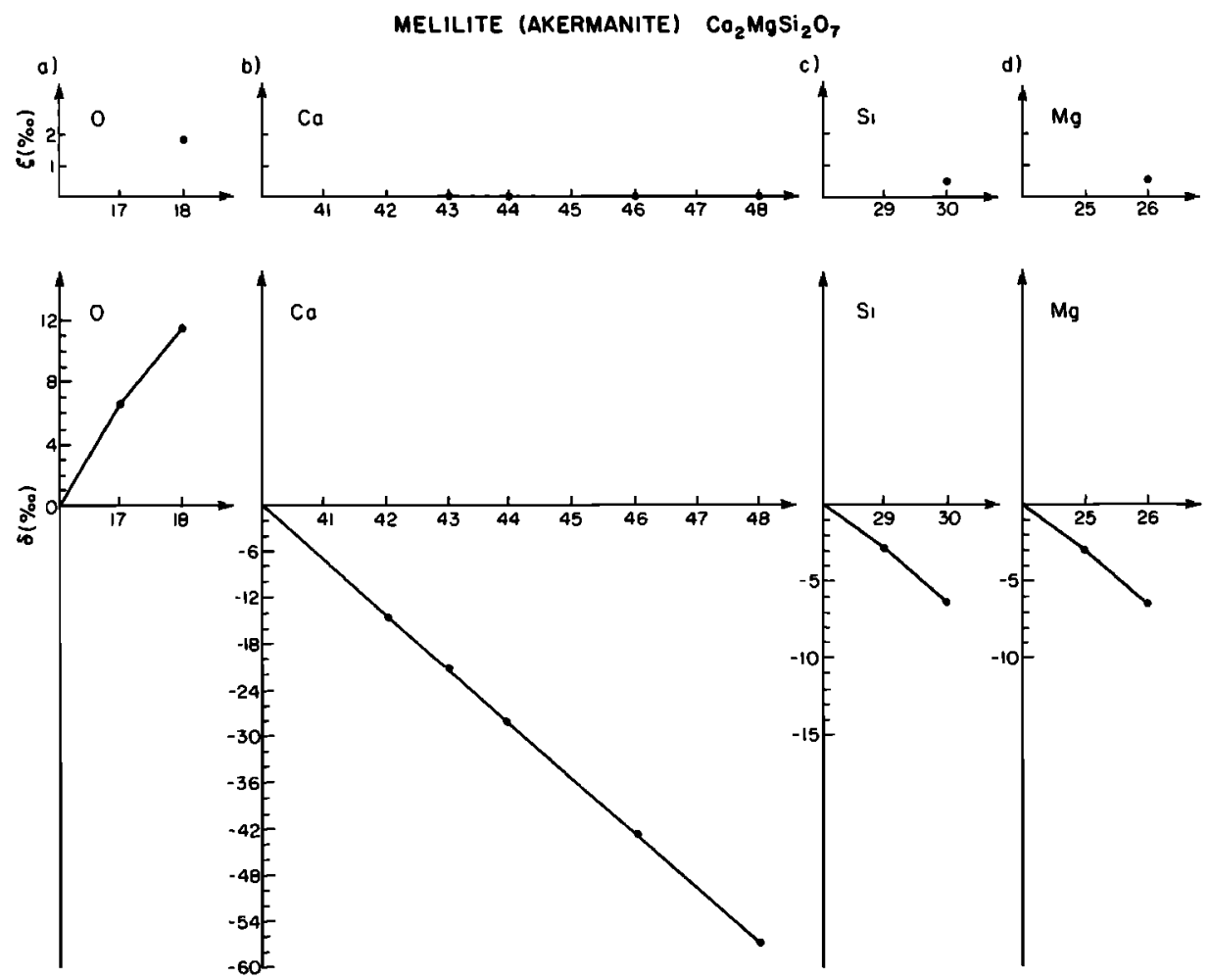

Fig. 8. In (a), (b), (c) and (d) the lower panels show the fractionation $\delta$ for $\mathrm{O}, \mathrm{Ca}, \mathrm{Si}$, and $\mathrm{Mg}$, respectively, for ackermanite, $\mathrm{Ca}_{2} \mathrm{MgSi}_{2} \mathrm{O}_{7}$. The upper panels in each case show the deviation from linearity as defined by (13) 
ENSTATITE MgSiO
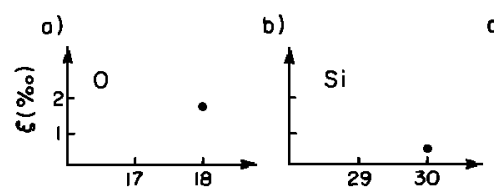

i $\mathrm{Mg}$
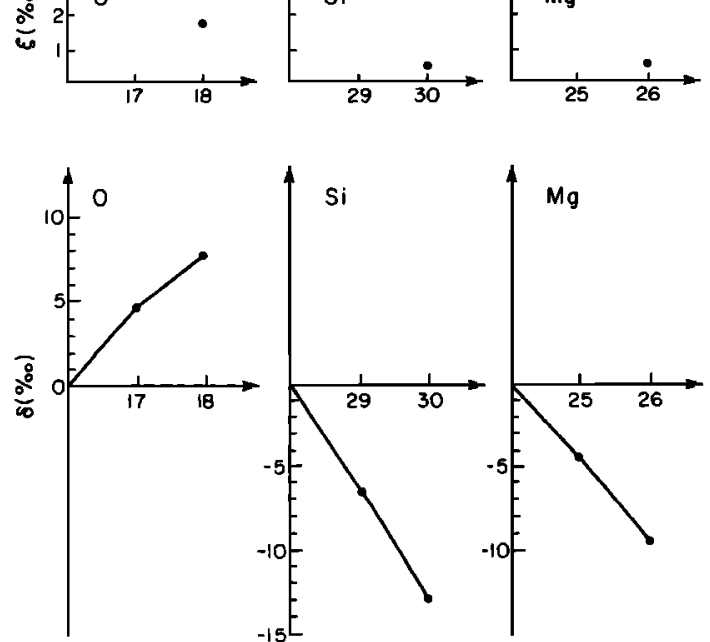

Fig. 9. In (a), (b), and (c) the lower panels show the fractionation $\delta$ for $\mathrm{O}, \mathrm{Si}$, and $\mathrm{Mg}$, respectively, in enstatite, $\mathrm{MgSiO}_{3}$. The upper panels in each case show the deviation from linearity as defined by (13).

same as for the $O$ component of quartz, Figure 5. In each mineral ${ }^{16} \mathrm{O}$ is the lightest component, and the coupling to the high mass components leads to preferential emission of the heavy isotopes. The effect is not a strong function of the mass of the heavy atoms, as long as they are reasonably abundant. In the $O$-containing minerals illustrated above, $O$ accounts for, to within a few percentage points, $60 \%$ of all atoms by number, while the heavier atoms, usually some combination of $\mathrm{Al}, \mathrm{Mg}, \mathrm{Si}$, and $\mathrm{Ca}$, make up the remaining $40 \%$. Thus $\mathrm{O}$ fractionation does not vary much from one mineral to the next. For similar reasons the heavier components of the mineral are in each case negatively fractionated, i.e., the lighter isotopes are sputtered preferentially.

The same kind of results are predicted for the non-oxygencontaining mineral troilite (FeS). $S$ is positively fractionated and Fe negatively fractionated, in both cases at the level of a few parts per thousand (ppt) per unit mass.

These seem to be the two hallmarks of sputter-induced isotopic fractionation in many minerals: (1) all isotopes are fractionated at the level of a few ppt per unit mass, independently of the precise mineralogy of the target, and (2) sputtered $O$ is isotopically heavy with respect to the $O$ in the target, while heavier sputtered elements $(\mathrm{Mg}, \mathrm{Si}, \mathrm{Ca}, \mathrm{Ti})$ are isotopically light. The magnitudes of the fractionations predicted are large compared to the precision of the most careful mass spectrometric analyses $(\sim 0.1 \%$ [ Lee, 1979]). The fact that sputtering of isotopes of the light and beavy elemental components of a given target is predicted to produce fractionations of different sign sharply distinguishes the kind of process described here from mechanisms based on thermal of diffusive kinetics, which can also lead to isotopic fractionation. Fractionation due to mechanisms such as these must be either positive or negative, but not both, for a given set of processed isotopes. The 'mixed' positive and negative fractionation patterns produced in this model would be suggestive signatures for the origin of any material that exhibited them.

Some of the illustrated fractionation curves exhibit, in addition, definite nonlinearities. The most regular and for the most part the largest of these is shown by the $O$ series. In every case studied, ${ }^{18} \mathrm{O}$ is less abundant in the sputtered material than a linear relation based on the $\delta(17,16)$ value would suggest. Alternatively, on the basis of the $\delta(18,16)$ value, we could say that the ${ }^{17} \mathrm{O}$ abundance is enhanced. Our procedure has been to compare all $\delta$ values predicted according to (2) with a straight-line value obtained by passing a line through the origin and through the $\delta$ value for the lightest pair $(M+\Delta, M)$ of stable isotopes. The upper panels in Figure 6-10 show the nonlinearities calculated by this scheme,

$$
\xi_{M+1} \equiv i \delta(M+\Delta, M)-\delta(M+i, M)
$$

The $\xi$ values for $\delta(18,16)$ are confined to the narrow range $1.8-1.9 \%$ and are essentially independent of the mineralogical matrix. Small nonlinear features also appear in some of the heavier isotopic series, but they are neither as regular nor generally as large as those found for $O$.

Before pursuing the possibilities inherent in a mechanism that can lead to anomalous isotopic patterns, however, we need to establish the validity of the model proposed here, and this can be done only by appeal to appropriate experiments. The present model arose out of the $\mathrm{Ca}$ fractionation data of Russell et al. [1980]. Watson and Haff [1980] found it necessary to invoke the surface flux model in order to obtain fractionation values of the magnitude required. But the model did not predict the experimental results; it can be said only to be consistent with them. However, the predicted positive-negative fractionation pattern illustrated in Figures 6-10 provides the kind of yes-no test that can give substantial support for or evidence against the proposed sputtering mechanism.

A test that seems to have merit involves measuring isotope ratios from the same chemical element in two different targets. The 'background' masses of the auxiliary partners would be different in the two cases and would be chosen to yield an expected positive fractionation of the isotopes of the given element in one case and a negative fractionation in the other. Because experience has already been gained in the measurement of fractionation of Ca-containing minerals under sputtering
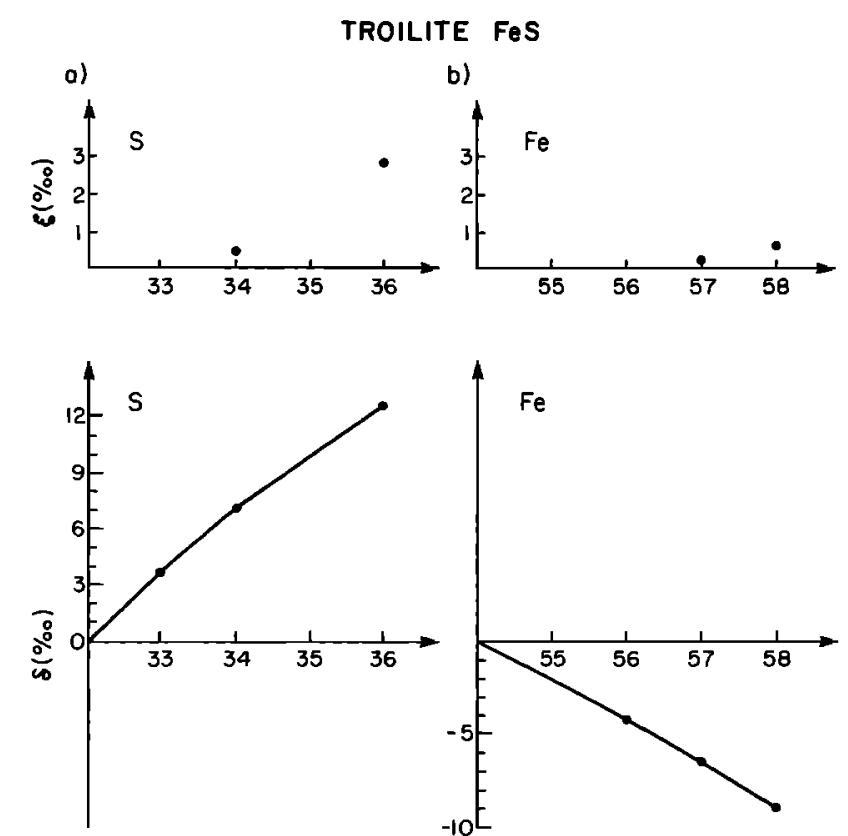

Fig. 10. In (a) and (b) the lower panels show the fractionation $\delta$ for $\mathrm{S}$ and $\mathrm{Fe}$, respectively, in troilite, FeS. The upper panels in each case show the deviation from linearity as defined by (13). 


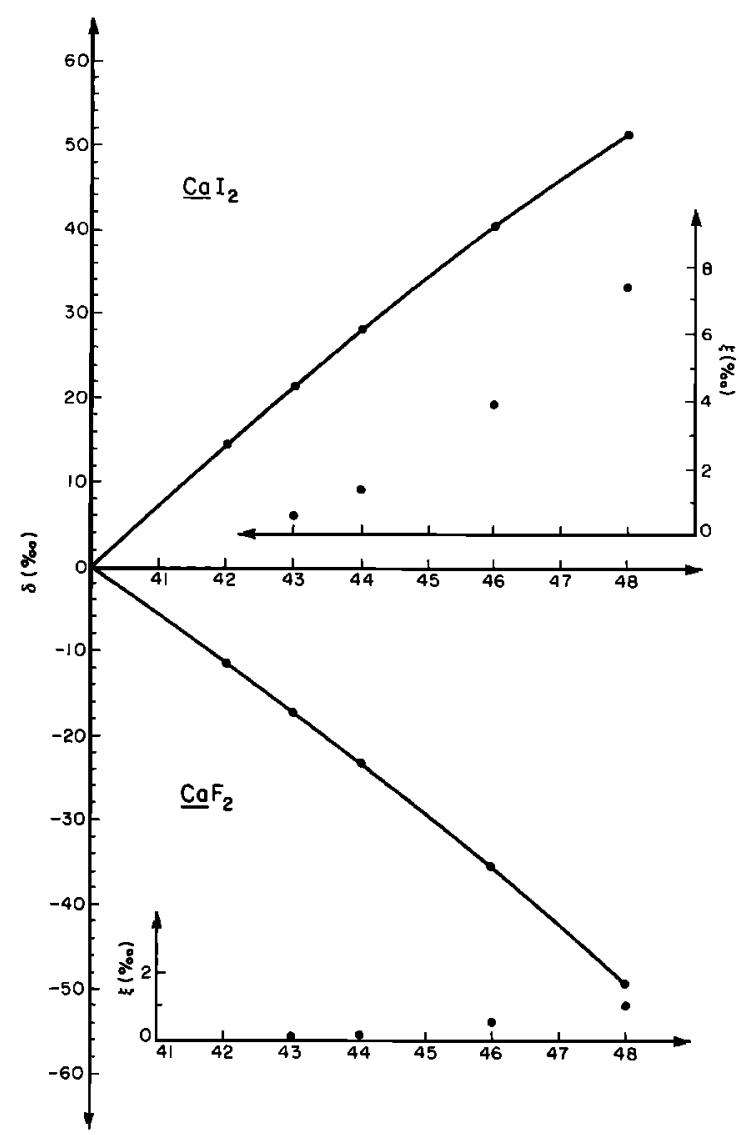

Fig. 11. The $\mathrm{Ca}$ fractionation curves for (top) $\mathrm{CaI}_{2}$ and (bottom) $\mathrm{CaF}_{2}$ are shown. The insets depict the deviation of these curves from a straight line through the origin and through the $\delta(42,40)$ point.

conditions [Russell et al., 1981], we examine predictions of (2) for two possible Ca-containing targets.

Figure 11 shows expected fractionations for fluorite, $\mathrm{CaF}_{2}$, and for $\mathrm{CaI}_{2}$. The elements $\mathrm{F}$ and $\mathrm{I}$ are each composed of a single stable isotope with mass 19 and 127 , respectively, and these two masses bracket the range of the $\mathrm{Ca}$ isotopes. The $\mathrm{Ca}$ sputtered from $\mathrm{CaF}_{2}$ is predicted to be strongly fractionated in a negative sense, and the $\mathrm{Ca}$ sputtered from $\mathrm{CaI}_{2}$ is predicted to be strongly fractionated in a positive sense. The magnitudes of the $\delta$ values are large, ranging from $\sim 10 \%$ to $\sim 50 \%$. Moreover, especially in the case of $\mathrm{CaI}_{2}$, the nonlinearities are also large, the maximum $\xi$ value exceeding $7 \%$. The direction of the nonlinearity is the same (positive) for both $\mathrm{CaF}_{2}$ and $\mathrm{CaI}_{2}$. This is also the case for the $\mathrm{Ca}$ metal fractionation curve illustrated in Figure 4. A positive $\xi$ value for a given isotope means that the isotope is more depleted in the sputtered material than a straight-line law based on the two lightest isotopes would suggest. Thus for $\mathrm{CaI}_{2}$ the enrichment of heavy isotopes is less than the value of $\delta(42,40)$ suggests, and for $\mathrm{CaF}_{2}$ the depletion of heavy isotopes exceeds a prediction based upon $\delta(42,40)$. This target pair could provide, then, an economical test of the sputtering model proposed here, since both the positive-negative fractionation feature as well as the nonlinearity characteristic can be investigated at the same time.

We conclude the presentation of this sputter-induced fractionation process by discussing some of the qualifications and difficulties that attend it. First, it must be emphasized again that the $\delta$ fractionation values apply strictly to the material that is sputtered away in the initial stages of bombardment, not to the surface material that is left behind. Furthermore, since $\delta$ depends upon the abundance of the various constituents at the surface (the $n_{l}$ in (3)), the amount of fractionation will change as sputtering proceeds. In principle, the $n_{i}$ 's should be considered to be functions of time, $n_{i}=n_{i}(t)$, and therefore $\delta=\delta(t)$. The total effective $\delta$ would then be obtained by integration. Unfortunately this is a complicated and uncertain procedure. The time dependence of $n_{t}(t)$ depends not only on the instantaneous partial sputtering yields of the various species, but it depends also upon how the material at the extreme outer surface is mixed with the underlying material. This mixing process [Haff and Switkowski, 1977] always accompanies ion bombardment in the region of a concentration gradient, and at the present time we are unable to treat the effect in a suitably quantitative manner. A rule of thumb that has been found useful is that preferential sputtering effects persist at decreasing levels until a thickness of material on the order of the range of the incident ion has been removed. For laboratory sputtering experiments using ions in the $\mathrm{keV}$ range this distance is quite small, on the order of $1700 \AA$ in the experiments of Russell et al. [1980]. These authors found, in fact, that $\delta$ values approached zero once $15-50 \%$ of the ion range had been sputtered away. The $\delta$ values quoted in this paper refer to the material removed in the very first moments of sputtering. Subsequent sputtering can be expected to lead to a dilution of the effect and hence to smaller effective $\delta$ values for the sputtered material. (Clearly $\delta \rightarrow 0$ rigorously when the target has been entirely sputtered away.) For this reason the $\delta$ values predicted here will tend to overestimate the corresponding measured quantity. Watson and Haff [1980] found that their predicted fractionation effects exceeded the measured values typically by a factor on the order of 2 .

It is also important to keep in mind that the surface flux model on which all the above results are based is an idealized and perhaps not totally consistent treatment of the effect that the introduction of a half-space type boundary can be expected to have on emission of particles from the surface. Thus if the true composition of the sputtered flux contains, in addition to the surface flux, a component arising directly from the (essentially stoichiometric) internal recoil cascade, then the predicted fractionation effects will be reduced.

Nevertheless, one can pose a rather definite test of these ideas, as exemplified by the $\mathrm{CaF}_{2}, \mathrm{CaI}_{2}$ system. A negative result in such an experiment would force a close reexamination of the surface flux model. A positive result would provide a significant stimulus to further investigations into the fractionation process. Especially interesting are problems that need to be addressed about the role of sputtering processes in space. Observed isotopic patterns in meteoritic inclusions provide important clues to the origin of the sun and planets. Since sputtering of grains by shock waves in the interstellar medium is likely to have occurred [Dwek and Scalo, 1979], we may ask how the resulting fractionations combine with those induced by nuclear reactions. The answer to such questions will depend upon the outcome of experiments designed to test fractionation theories such as those presented here.

Acknowledgments. PKH would like to thank W. A. Fowler and G. J. Wasserburg for focussing his attention on sputtering processes in mineral assemblages. Helpful comments from D. S. Burnett and D. A. Papanastassiou have been incorporated in the text. This work was supported in part by the National Aeronautics and Space Administration (grant NGR-05-002-333) and by the National Science Foundation (grant PHY79-23638). 


\section{REFERENCES}

Clayton, D. D., Origin of Ca Al-rich inclusion, 2, Sputtering and collisions in the three-phase interstellar medium, Astrophys. $J$. , in press, 1981.

Dwek, E., and J. Scalo, Interstellar depletions and the filling factor of the hot interstellar medium, Astrophys. J. Lett., 233, L81-L85, 1979.

Grossman, $\mathrm{L}$., Condensation in the primitive solar nebula, Geochim. Cosmochim. Acta, 36, 597-619, 1972.

Haff, P. K., and Z. E. Switkowski, On the sputtering of binary compounds, Appl. Phys. Lett., 29, 549-551, 1976.

Haff, P. K., and Z. E. Switkowski, Ion-beam-induced atomic mixing, J. Appl. Phys., 48, 3383-3386, 1977.

Lee, T., New isotopic clues to solar system formation, Rev. Geophys. Space Phys., 17, 1591-1611, 1979.

Liau, Z. L., W. L. Brown, R. Homer, and J. M. Poate, Surface layer composition changes in sputtered alloys and compounds, Appl. Phys. Lett., 30, 626-628, 1977.

Russell, W. A., D. A. Papanastassiou, and T. A. Tombrello, The fractionation of calcium isotopes by sputtering, Radiat. Eff., 52, 41-52, 1980.
Sigmund, $P$. , Theory of sputtering, 1, Sputtering yield of amorphous and polycrystalline targets, Phys. Rev., 184, 383-416, 1969.

Switkowski, Z. E., P. K. Haff, T. A. Tombrello, and D. S. Burnett, Mass fractionation of the lunar surface by solar wind sputtering, $J$. Geophys. Res., 82, 3797-3804, 1977.

Thompson, M. W., The energy spectrum of ejected atoms during the high energy sputtering of gold, Philos. Mag., 18, 377-414, 1968.

Watson, C. C., Topics in classical kinetic transport theory with applications to the sputtering and sputter-induced mass fractionation of solid surfaces and planetary atmospheres, $\mathrm{Ph}$.D. thesis, Yale Univ., New Haven, Conn., 1980.

Watson, C. C., and P. K. Haff, Sputter-induced fractionation at solid surfaces, J. Appl. Phys., 5I, 691-699, 1980.

Weller, R. A., and T. A. Tombrello, Energy spectrum of sputtered uranium-A new technique, Radiat. Eff., 37, 83-92, 1978.

(Received March 2, 1981;

revised June 5, 1981;

accepted June 5, 1981.) 